\title{
Candidate Selection for the FLAMINGOS-2 Galactic Center Survey
}

\author{
Curtis DeWitt, Stephen Eikenberry, and Reba Bandyopadhyay \\ Department of Astronomy, University of Florida, 211 Bryant Space Science Center, \\ Gainesville, FL 32611 \\ Robert Blum \\ NOAO, 950 North Cherry Ave. Tucson, AZ 85719
}

Michael Muno

Department of Astronomy, Caltech, MC 105-24, 1200 East California Blvd, Pasadena CA 91125

Kris Sellgren

Department of Astronomy, Ohio State University, $140 \mathrm{~W}$. 18th Avenue, Columbus, OH 43210

\begin{abstract}
We present a $J H K_{s}$ catalog of a 20' x 20' region around the Galactic Center observed with the ISPI camera on the $4 \mathrm{~m}$ CTIO telescope. The data is being used to select targets for the FLAMINGOS-2 Galactic Center Survey, which will take near-infrared spectra of thousands of GC sources in an effort to identify and characterize the unique X-ray binary source population in this region.
\end{abstract}

\section{Introduction}

The inner $150 \mathrm{pc}$ around the Galactic Center contains a population of 2000 faint $\left(L_{x}<\right.$ $10^{34} \mathrm{erg} \mathrm{s}^{-1}$ ) and spectrally hard X-ray sources (Muno et al. 2006). These stars are likely to be a mix of old cataclysmic variables and accreting wind binaries. The fields are extremely crowded, however, and many seemingly likely IR counterparts will be chance superpositions. IR spectra can find accretion signatures characteristic of X-ray binaries and sort out the true counterparts. FLAMINGOS-2 is a near-infrared multi-object spectrograph ideally suited to pursue this task and this is the primary driver of the FLAMINGOS-2 Galactic Center Survey.

\section{Catalog Characteristics}

The data was taken in August 2005 at the CTIO 4m telescope. We used the infrared camera ISPI to take J, H, and Ks imaging of the 20'x20' field. The $5 \sigma$ limiting magnitudes for our point sources were $J=21.6, H=17.5$ and $K=17.1$. We used the standalone version of DAOPHOT to do the source detection and photometry. The catalog contains 51,000, 172,000 , and 176,000 sources in $\mathrm{J}, \mathrm{H}$, and $K_{s}$ bands, respectively. Crowding is extremely severe, causing many sources to be blended and miss detection. We are performing artificial 

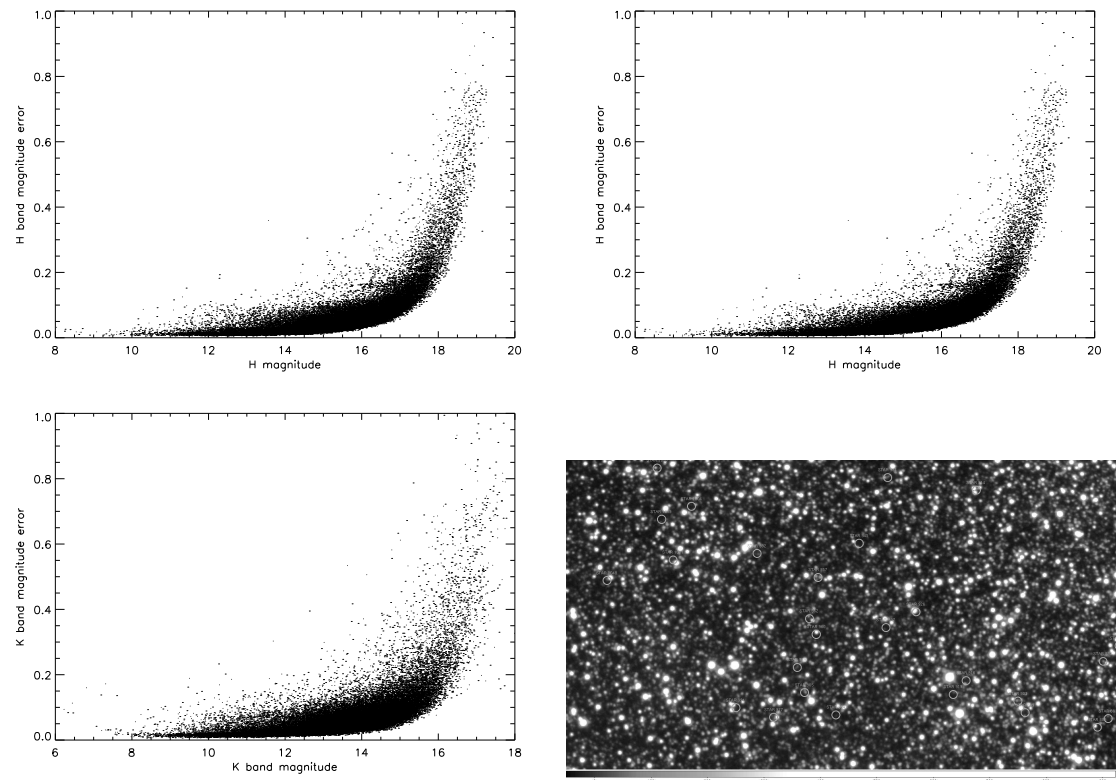

Figure 1. Panels 1-3: Percent error versus magnitude. Panel 4: A section of the GC field showing X-ray error circles overlaying the $\mathrm{K}$ band ISPI data.

star tests now to quantify the completeness limits of our catalog. The photometric error peaks at $1 \%$ for all three bands and falls off in the expected manner from photon noise. We used the 2MASS catalog to find the photometric zeropoints. The RMS scatter of the zeropoint was about 0.1 magnitudes in all three bands. Astrometry was also tied to 2MASS using the program "tfinder" in IRAF. The RMS scatter in the astrometry was 0.3 arcsec. We matched the J, H and $K_{s}$ catalogs together with the program TOPCAT. We used 0.3 arcsec, the astrometric scatter in the frame tie, as our match criterion. We find that $73 \%$ of $\mathrm{J}$ band sources have an $\mathrm{H}$ band counterpart and $55 \%$ have both $\mathrm{H}$ and $K_{s}$ counterparts. $72 \%$ of $\mathrm{H}$ band sources have a $K_{s}$ band counterpart. The large number of unmatched $\mathrm{J}$ sources is due to faint foreground stars picked up by the $\mathrm{J}$ band limit, but missed in the shallower $\mathrm{H}$ and $K_{s}$ band exposures.

\section{Infrared Counterparts to Chandra Sources}

We used TOPCAT again to match the X-ray source positions (Muno et al. 2006) to the ISPI data sources to find potential counterparts. This time the match criterion was the combined astrometric uncertainty in the infrared data and the X-ray catalog's positional uncertainty, (usually on the order of an arcsec). $68 \%$ of X-ray sources have at least one $K_{s}$ and $\mathrm{H}$ source within the error circle. $10 \%$ of these have an additional potential match within the error circle. Only $85 \%$ of matches are expected to be true (Eikenberry 2007). 


\section{Future Work}

We are currently simulating more robust error estimates and completeness fractions with artificial star tests in DAOPHOT. We will also construct a fine-grained extinction map of the region using the technique developed by Andrew Gosling (Gosling et al. 2006), and deredden the sources so that we can analyze the X-ray counterparts and field star environments. We will select targets and design the slit masks from the completed catalog and in summer 2008, we will conduct the spectroscopic survey of the counterparts with FLAMINGOS-2.

Acknowledgments. C. DeWitt thanks Craig Warner for assistance with his PYRAF pipeline, FATBOY, used for the image reduction in this work.

\section{References}

Eikenberry, S. 2007, RMxAC, 29, 143

Gosling, A. J., Blundell, K. M., \& Bandyopadhyay, R.M. 2006, ApJ, 640, L171

Muno, M.P., Bauer, F.E., Bandyopadhyay, R.M., Wang, Q.D. 2006, ApJS, 165, 173 